\title{
Management and Complications of Nasal Septal Collections
}

\author{
Olusola Ayodele Sogebi, Emmanuel Abayomi Oyewole \\ ENT Unit, Department of Surgery, Faculty of Clinical Sciences, OACHS, Olabisi Onabanjo University, \\ Sagamu, Nigeria
}

Correspondence to: Dr. Olusola Sogebi; email: ayosogebs@gmail.com

Received: 29 May 2020; Revised: 24 July 2020; Accepted:11 August 2020; Available online: 1 September 2020

\begin{abstract}
Background: Nasal septum collections (hematoma and abscess) can lead to structural and functional abnormalities. Our objective was to assess the clinical characteristics, management and complications of nasal septal collections, and document factors associated with their complications. Methods: This was a retrospective study of patients managed for nasal septal collections. Socio-demographic and clinical information was recorded, and the main investigations and results noted. Follow-up and complications of septal collections were documented and the clinical factors associated with the complications explored. Results: Twenty-four patients records were studied: male: female ratio $=2: 1$, mean age $40.1 \pm 13.1$ years, $62.5 \%$ presented with complaints of nasal obstruction, $66.7 \%$ had antecedent nasal trauma, presentation was from 2 to 13 days, $25 \%$ had co-morbid disease(s). All patients had incision and drainage of the septal collection within $1-7 \mathrm{~h} ; 41.2 \%$ of the aspirated collections cultured microorganisms, $20.8 \%$
\end{abstract}

developed complications. Increased age above 45 years, co-morbidity, delayed presentation, culture-positive aspirate was all significantly associated with development of complications. Conclusion: Nasal septal collections were more common in adult males with antecedent nasal trauma; 20\% developed complications associated with the presence of culturepositive abscesses, increased age, and duration of septal collection.

Keywords: Nasal trauma, Septal hematoma, Septal abscess, Complications

Ann Afr Surg. 2021; 18(2): 79-84

DOI: http://dx.doi.org/10.4314/aas.v18i2.4

Conflicts of Interest: None

Funding: None

(C) 2021 Author. This work is licensed under the Creative Commons Attribution 4.0 International License.

\section{Introduction}

Nasal septal collection is an accumulation of fluid between the cartilage or the bony septum and the overlying mucoperichondrium or mucoperiosteum (1). The fluid is either blood (hematoma) or pus (abscess). Septal collections are generally not common; the prevalence of nasal septal hematoma and abscess in children has been reported as $0.9 \%$ (2). Many publications on septal collections have been on hematomas while studies that combined septal collections reported comparatively more hematomas than abscess $(3,4)$. Oftentimes an abscess results from a secondary infection of hematoma (5), thus it is rarer. Most reports of septal abscess involve adults who had experienced nasal trauma (1).

The main etiology of septal collections is different types of trauma to the nose. The mechanism that induces a septal hematoma is a buckling stress that tears the submucosal blood vessels. If the mucosa remains intact, the blood will accumulate between the mucoperichondrium and the septal cartilage, which 
Management and Complications of Nasal Septal Collections

relies on the mucoperichondrium for its blood supply. The stagnant blood is an excellent medium for bacteria to proliferate and it eventually results in the formation of a localized abscess (6). Common types of trauma include explosive forces, leading to fracture of nasal bones in road traffic accidents, blows from punches, and sports injuries to the nose (7). Sometimes infections in contiguous areas of the face such as paranasal sinuses, oral cavity, or orbits $(6,8)$ lead to septal collection. Spontaneous septal abscess has been associated with immunocompromised disease conditions $(5,9)$.

Appropriate management of a nasal septal collection requires prompt diagnosis, adequate surgical drainage, and parenteral administration of antibiotics (1). However, complications may arise when the accumulation has remained for a relatively long period or an abscess has developed. Thus, optimal time is required in the treatment to have a good outcome and prevent complications (10). The dreaded complications include severe functional and cosmetic sequelae with deformity of the nasal bridge, forming a saddle nose (11). The goals of management will include relief of nasal obstruction, maintenance of the nasal bridge architecture, and prevention of intracranial complications.

The medical literature on septal collections has been mostly case reports about strange or unusual causes of septal collections, whereas the characteristics and profile of septal collections has been less reported. Factors associated with complications have rarely been sought or reported. This study aimed to assess the characteristics, management and complications of nasal septal collections, and to also explore clinical factors that may be associated with the complications. This will suggest the factors to explore in cases of septal collections to accomplish the best outcome possible.

\section{Methods}

This was a retrospective cross-sectional study of patients who were admitted and managed at the Ear, Nose and Throat Department of a teaching hospital, in southwestern Nigeria. The patients were managed within an 8-year period, from January 2012 to December 2019. The study protocol was approved by the Institutional
Health Research Ethics Committee, approval number 341/2020AP. Eligible patients had nasal septal collection (either hematoma or abscess) and were managed within the study period. The patients excluded were those who had associated intracranial injury or infection and those referred to other medical centers for primary management. Records that had missing important information of patients were also excluded.

The case note records of the patients were retrieved from the clinic, emergency center, and ward admission registers to obtain clinical information. Information extracted included socio-demographic parameters such as age and sex, clinical information for the main presenting symptom(s), duration of symptom(s) before presentation at the hospital, predisposing factors, presence of co-morbid disease or otherwise. The main investigations were noted and the results of microscopy culture and the sensitivity of the aspirate of the septal collection were recorded.

The protocol employed was the standard procedure for surgically draining nasal septal hematoma and abscess described by Kass and Ferguson (12). Topical administration of a mixture of $2 \%$ lidocaine for local anesthesia and oxymetazoline solution (to constrict the nasal turbinates) were used to obtain a clearer view. A Jshaped incision was made on the mucosa of the bulgy side of the septal collection and deepened into the cavity. Fluid was directly aspirated with a syringe to collect a sample for microbiological analyses. Suction drainage with a machine completed evacuation of the cavity. Both nasal cavities were packed firmly with lubricated antibiotic-laden gauze to prevent or reduce the tendency of the fluid to re-accumulate. We routinely administered parenteral antibiotics during and continued for a minimum of $24 \mathrm{~h}$ after the drainage procedure. The antibiotics regimen was reviewed based on the microscopy, culture, and sensitivity results of the aspirate. Nasal packs were usually removed after $72 \mathrm{~h}$. A few modifications such as irrigating the septal cavity with $0.9 \%$ saline in septal abscess or use of a tampon for nasal packing were made as the situation demanded.

The follow-up duration (based on the last record) was calculated, and the outcome and complications of the septal collections were noted. 
Data generated were presented in a general descriptive format while comparative analyses of clinical factors associated with development of complications used SPSS version 21.0 (Chicago, IL, USA). Categorical variables were compared using the chi-square test. Normality of continuous variables was explored using the Shapiro-Wilk test, and comparative analyses used Student's $t$-test. The results were presented in tabular format with $\mathrm{p}<0.05$ considered statistically significant.

\section{Results}

Thirty-one patients were managed for nasal septal collection during the study period, and 24 case records with complete information were retrieved. There were 16 male and 8 female patients, male: female ratio $=2: 1$. The ages ranged from 13 to 62 years, and the highest proportion of patients were in the 21-40 years age group. Age group distribution according to sex of the patients revealed no females in the $<20$ years age group, male preponderance was observed in other age groups (21-40 and 41-60 years), up to the 61 years and above group that had one male and one female. The mean age was $40.1 \pm 13.1$ years. Patients presented with varying complaints: $62.5 \%$ presented with the main complaint of nasal obstruction or blockage, and $29.2 \%$ with nasal swelling. Two-thirds (66.7\%) of the patients had a history of nasal trauma before developing the collections while there was no obvious etiology in five (20.8\%) patients. All patients presented between 2 and 13 days from the onset of symptoms/predisposing factors, with a median of 4 days. Three-quarters of the patients had no co-morbid disease with the nasal septal collections, while three (12.5\%) had uncontrolled diabetes.

All patients had the septal collections incised and drained. The waiting time of the patients before the procedure ranged from 1 to $7 \mathrm{~h}$ (median 4). All patients had aspirates of the collection sent for microscopy, culture, and sensitivity. Seventeen (70.8\%) patients had documented aspirate microbiological results, with seven $(41.2 \%)$ reporting the growth of microorganisms. The organisms cultured were Staphylococcus aureus in five patients and Klebsiella pneumonia and Streptococcus pneumonia in one patient each.
The final diagnosis of the patients was septal hematoma in $15(62.5 \%)$ and abscess in 9(37.5\%). Follow-up was for a minimum of 6 months; the range was 6-15 (median 9) months. Five patients (20.8\%) developed complications including nasal deformity in three patients, facio-orbital cellulitis in one patient, and intracranial infection in one patient.

Clinical parameters were compared between patients who did not have and those who had complications, to explore the factors associated with the complications (Table 1). In the comparative analyses, any clinical factor with $\mathrm{p}<0.05$ (statistically significant) was associated with complications of septal collection. Increased age especially above 45 years, presence of a co-morbidity, longer duration before presentation, presence of an abscess, and a culture-positive aspirate were all significantly associated with complications of nasal septal collections.

\section{Discussion}

The nose is the most prominent facial structure. Only a small percentage of patients with nasal trauma will develop a septal hematoma (13). Nasal septal collections can thus be described as an uncommon sequalae of nasal trauma. While some studies have reported nasal septal collections to be more common in children (4), this study found almost a total adult occurrence of $95.8 \%$. Cheng et al. also reported a preponderance of adults in a study on septal abscesses (11). Septal collections were twice as common in males than in females in this study, similar to what was reported in other studies $(3,14)$. A previous study however reported no sex preponderance with septal abscess (11).

The nasal bone is the most commonly fractured bone in the human body (15). Thus, nasal trauma is by far the most common cause of septal collections, as it is estimated that nearly $75 \%$ of cases were secondary to trauma (5). Two thirds (66.7\%) of the patients evaluated in this study had antecedent nasal trauma which predisposed them to a septal hematoma, as seen in $62.5 \%$ of our patients. While common causes and mechanisms of nasal trauma were mentioned, one of our 


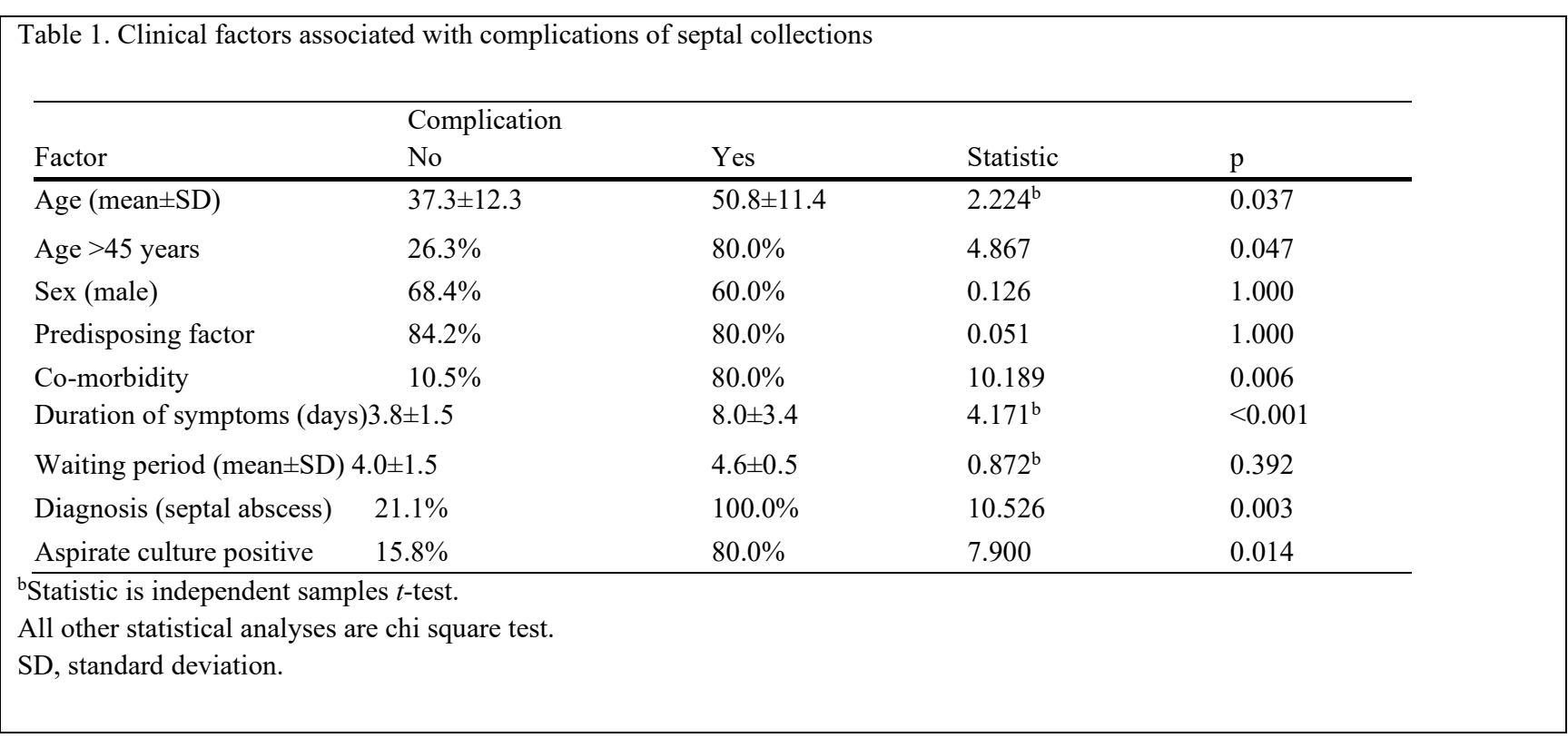

patients had an unusual etiology of domestic violence while she was pregnant. As nasal trauma can sometimes lead to septal hematomas, any patient presenting with acute nasal obstruction after facial or nasal trauma should be a suspect for a septal hematoma (16). Sometimes hematoma formation may be delayed after a nasal trauma, thus re-evaluation for septal hematoma 48 to $72 \mathrm{~h}$ after nasal trauma is recommended (17).

Septal abscesses are rarely seen, as they are often complications of hematoma from a secondary infection (5). One major predisposition to abscess formation is delayed or prolonged accumulation of blood in the nasal septum. Clinicians have advocated early drainage of septal collections, as further delay increases the probability of abscess formation and a tendency to develop complications (18). The median duration of presentation of our patients was 4 days, and the propensity to develop an abscess was theoretically high. The major presenting symptoms of nasal obstruction and blockage found in this study were similar to those reported by other authors. While this finding is almost pathognomonic of a septal collection, septal tumor or other forms of chronic granulomatous infections can present in a similar manner $(19,20)$.

The diagnosis of nasal septal collection is largely by clinical evaluation as investigation may be limited to aspirating the collection for bacteriological evaluation. The main treatment was incision and drainage of the septal collection. Surgical drainage should be performed as soon as possible because a delay may affect the outcome of the procedure (16). The median duration of waiting before drainage of the septal collections in this study was $4 \mathrm{~h}$.

All septal aspirated fluids were examined for bacteria; although results could not be found in seven patients, seven others had culture-positive results. The main microorganism cultured was indigenous skin bacteria, $S$. aureus, in $71.4 \%$ of the culture-positive specimens. Other studies $(6,21)$ also reported similar findings. Less frequently, some species of Streptococcus, Klebsiella, and occasionally anaerobes were cultured $(6,11)$. We did not get any positive culture for anaerobes despite our request. Similarly, there was no culture of methicillinresistant $S$. aureus (MRSA), although this was not specifically requested for. The expanding spectrum of severe infections caused by MRSA have been noted $(1,22)$. It has thus been suggested that patients who are at increased risk for MRSA colonization should be administered antibiotics against MRSA initially (11). MRSA is also associated with severe complications of nasal collections. Toxic shock syndrome is another lifethreatening systemic condition associated with Staphylococcus infection (23), but it was not found in this study.

The complication rate of $20.8 \%$ reported was relatively high. All patients that developed complications had 
septal abscess as their final diagnoses. The main complication was nasal bridge deformity in $33.3 \%$, while one patient each $(11.1 \%)$ among the nine patients with septal abscess developed facio-orbital cellulitis and intracranial infection. This is similar to the $33.3 \%$ of cases of septal abscess that developed saddle-nose deformity reported in Taiwan (11). Factors predisposing to the development of saddle-nose deformity include a septal perforation at surgery, resorption of the septal cartilage from loss of vascular supply, infection, or a combination of these (24). Our patients who had nasal bridge deformities were co-managed with the plastic surgeon for a septoplasty. One patient developed a facioorbital cellulitis and was treated with parenteral antibiotics in addition to other supportive treatments. One patient developed an intracranial infection 13 days after the initial draining of the septal abscess and was referred to a neurosurgical facility.

Exploration of factors associated with the development of complications provided insights for management considerations. Notably, increased age may be linked with the presence of co-morbidities. Two of our patients were diagnosed with diabetes after the septal collections had been incised and drained. Uncontrolled diabetes has been noted as an immunocompromised condition that may predispose patients to develop a spontaneous septal abscess (11). Thus, it is imperative that patients with a septal abscess should, among other investigations, have their blood sugar profile checked. Retroviral screening should also be performed on such patients for the same reason. One of our patients was retroviral positive and was managed accordingly.

Delayed presentation is associated with complications because irreversible damage to the septum with a subsequent septal perforation may occur within 3 to 4 days if it is devoid of its blood supply (24). Some of our patients were already at risk of developing complications by the time they presented at the hospital. Septal abscesses, especially those that were culture positive, had active bacterial infection within the nose, which could easily drain through the veins into the face and intracranially. Consequently, any diagnoses of a septal abscess should also be treated aggressively with appropriate parenteral antibiotics for a reasonable period. Although drainage of nasal septal abscess with concomitant antibiotic administration prevents early complications, it is not enough guarantee for satisfactory functional and cosmetic effect in the future (2).

Satisfactory surgical outcome is guaranteed if there are no late complications, thus patients must be followed up for a reasonable period of time. Complications like saddle-nose deformity may not be recognizable until a few months after the nasal and facial swellings have completely subsided. We followed up our patients for a minimum of 6 months after the septal collections had been drained. Our standard follow-up protocol emphasized the possibility of recurrence or complications, and stressed to patients the necessity and importance of keeping follow-up appointments. The home and telephone contacts of the patients were collected, and the surgeon's number given to the patients for communication. With this we were able to achieve a reasonable follow-up course.

Despite our management protocol, some limitations were obvious in this study. The retrospective nature of the study had an inherent tendency for loss and missing information or data. The fact that patients' clinical case records were assessed during the study made it difficult to perform an objective (rather than subjective) functional assessment of nasal performance. However, these limitations did not invalidate the findings of the study.

\section{Conclusion}

Nasal septal collections were often hematomas and were more common in adult males with antecedent nasal trauma. All patients were managed by surgical drainage and administration of antibiotics. One-fifth developed complications, especially nasal bridge deformity. Factors associated with complications were increased age, presence of co-morbidity, longer duration of septal collection before drainage, and septal abscess particularly in those who were culture positive.

\section{Acknowledgments}

The authors wish to acknowledge the efforts of Dr Taofeeq Mabifah for assisting in retrieving some of the case note records of patients that were studied. 


\section{References}

1. Huang Y, Hung P, Lin H. Nasal septal abscess in an immunocompetent child Pediatr Neonatol. 2012;53(3):213-215.

2. Zielnik-Jurkiewicz B, Olszewska-Sosińska O, Rapiejko P. Treatment of the nasal septal hematoma and abscess in children. Otolaryngol Pol. 2008;62(1):71-75.

3. Sayin I, Yazici ZM, Bozkurt E, et al. Nasal septal hematoma and abscess in children. J Craniofac Surg. 2011;22(6): e17-19.

4. Nwosu JN, Nnadede PC. Nasal septal hematoma/abscess: management and outcome in a tertiary hospital of a developing country. Patient Prefer Adherence. 2015; 9:1017-1021.

5. Chung JC, Wong AT, Ho W. Spontaneous nasal septal abscess presenting as complete nasal obstruction. Int $\mathrm{J}$ Otolaryngol Head Neck Surg. 2013; 2:79-81.

6. Fatima SN, Sarwar F, Khan MS. Nasal septal abscess as a sequela of orbital cellulitis: an uncommon presentation. SAGE Open Medical Case Rep. 2018; 6:1-3.

7. Marston AP, O'Brien EK, Hamilton GS 3rd. Nasal injuries in sports. Clin Sports Med. 2017; 36:337-353.

8. Kop EA, Lodder WL, de Visscher JG, et al. Nasal septal abscess caused by protrusion of a dental implant into the nasopalatine duct: A case report. Ann Otol Rhinol Laryngol. 2020; 129:633-636.

9. Wang Y, Chen HC. Spontaneous nasal septal abscess. J Emerg Med. 2019;57: e131-132.

10. Wang W, Lee T, Kohlert S, et al. Nasal fractures: The role of primary reduction and secondary revision. Facial Plast Surg. 2019; 35:590-601.

11. Cheng LH, Wu PC, Shih CP, et al. Nasal septal abscess: A 10-year retrospective study. Eur Arch Otorhinolaryngol. 2019; 276:417-420.

12. Kass JI, Ferguson BJ. Treatment of hematoma of the nasal septum. N Engl J Med. 2015;28;372: e28.

13. Puricelli MD, Zitsch RP 3rd. Septal hematoma following nasal trauma. J Emerg Med. 2016; 50:121-122. 